\title{
Measurement of phagocyte activity in heterotherms
}

\author{
Tomas Heger ${ }^{1}$, Jan Zukal ${ }^{2,3}$, Veronika Seidlová ${ }^{1}$, Monika Nemcova ${ }^{1}$, David Necas ${ }^{4}$, \\ Ivana Papezikova ${ }^{1}$, Vladimir Piacek ${ }^{1}$, Renata Zajickova ${ }^{2}$, Hana Bandouchova ${ }^{1}$, Jiri Pikula ${ }^{1}$ \\ ${ }^{1}$ University of Veterinary and Pharmaceutical Sciences Brno, Faculty of Veterinary Hygiene and Ecology, \\ Department of Ecology and Diseases of Zoo Animals, Game, Fish and Bees, Brno, Czech Republic \\ ${ }^{2}$ Czech Academy of Sciences v.v.i., Institute of Vertebrate Biology, Brno, Czech Republic \\ ${ }^{3}$ Masaryk University, Faculty of Science, Department of Botany and Zoology, Brno, Czech Republic \\ ${ }^{4}$ Masaryk University, CEITEC, Plasma Technologies, Brno, Czech Republic
}

Received October 24, 2019

Accepted February 25, 2020

\begin{abstract}
The heterotherm immune system undergoes significant variation in response to life cycle periodicity and torpor. As heterothermic bats are important reservoirs of zoonotic agents and modulation of immune activity can affect host-pathogen interactions, this work aimed at developing a suitable method for assessing heterotherm phagocyte activity. Chemiluminescence measurements were evaluated by mathematical and mechanistic approaches, both of which yielded comparable results in time-related parameters of phagocyte activity. Using a mathematical method, however, we developed a model that can be applied to particular specimens. The proposed equation offers a simple and reliable tool for comparing phagocyte activity, the values of which can be used for further analysis. While time-related parameters of bat phagocyte activity varied with measurement temperature, with the onset of respiratory burst at $38^{\circ} \mathrm{C}$ being quicker than at $25^{\circ} \mathrm{C}$, quantitative values of phagocyte activity were not influenced by measurement temperature. Further, homeotherm phagocyte activity parameters were more variable at $25{ }^{\circ} \mathrm{C}$. Considering there was no influence of measurement temperature on the total volume of heterotherm phagocyte activity, we suggest that parameters measured at $25{ }^{\circ} \mathrm{C}$ are more representative of the immune status adapted to physiological extremes at low body temperatures.
\end{abstract}

Innate immunity, respiratory burst, torpor, hibernation, bat, laboratory mouse

The immune system of heterothermic mammals is greatly influenced by energyconserving mechanisms such as hibernation and daily torpor (Bouma et al. 2010a; Bouma et al. 2010b; Bouma et al. 2013). The efficiency of immune responses is speciesspecific and depends on the temperature range utilised (Andjus et al. 1964; Marnila et al. 1995; Lojek et al. 1997; Mondal and Rai 2001; Maniero 2002; Buchtikova et al. 2011), with phagocyte activity and other immune functions being reduced significantly by decreased body temperatures (Carey et al. 2003; Bouma et al. 2010a).

The most pronounced change in the immune system of heterotherms is a reduction in the number of circulating phagocytic cells (Bouma et al. 2010a). The decrease in the number of circulating neutrophils during torpor could be explained by lowered production of neutrophils, increased apoptosis, adherence of cells to the endothelium of mesenteric venules and temporary retention of cells at specific locations, such as the lungs, liver and spleen (Wils on et al. 1958; Inkovaara and Suomalainen 1973). According to Bouma et al. (2013) neutrophils are retained in the marginal pool (i.e. adherent to the vessel wall) during torpor and released during arousal. Circulating phagocytic cells use reactive oxygen species (ROS) for killing pathogens. ROS are toxic substances that display microbicidal activity against pathogens engulfed by phagocytes (Murphy et al. 2012). This granulocyte reaction is termed respiratory burst (RB) and is quantified using a chemiluminescence assay.

Address for correspondence:

Jiri Pikula, Tomas Heger

Department of Ecology and Diseases of Game, Fish and Bees

University of Veterinary and Pharmaceutical Sciences Brno

Palackého tř. 1946/1, 61242 Brno, Czech Republic 
This method has previously been used to measure RB in both homeothermic wistar rats and poikilothermic frogs and fish (Marnila et al. 1995; Lojek et al. 1997; Buchtikova et al. 2011) but, to our knowledge, has never been applied to heterothermic mammals.

Hibernation and daily torpor are multi-stage events during which heterotherms undergo sequential physiological stages. Periodicity in torpor/arousal cycles includes euthermia, cooling into torpor, prolonged torpor and re-warming from torpor. Each stage involves varying metabolism and immune system states, each of which is of species-specific duration (Boyer and Barnes 1999; Heldmaier et al. 2004; Havenstein et al. 2016).

The aim of this study was to elaborate a method for evaluating heterotherm phagocyte activity that would be useful for analysing the highly variable physiological states undergone during hibernation. Based on present knowledge of the bat immune system, and the argument that hibernation is not a uniform state, we also assessed (1) differences between heterotherms (two bat species) and homeotherms (laboratory BALB/C mouse Mus domesticus), and (2) differences induced by the temperature at which phagocyte activity is measured. We hypothesise that heterothermic bats display greater variability in the quantitative parameters measured, mirroring their physiological states during hibernation/daily torpor, and that lower measuring temperatures delay the onset of the chemiluminescence curve and reduce peak phagocyte activity.

\section{Materials and Methods}

Eleven cave-hibernating bats (greater mouse-eared bat Myotis myotis) and 12 rock-crevice and/or tree-hole hibernating bats (common noctule Nyctalus noctula) were sampled soon after the hibernation period in 2018 . To collect blood from the uropatagial vessel, the bats were manually restrained and the overlying skin was disinfected with alcohol. Blood (approximately $120 \mu \mathrm{l}$ ) was then drawn from a sterile needle puncture site using a heparinised pipette tip (Pikula et al. 2017; Bandouchova et al. 2018). Blood was collected from the saphenous vein of 14 adult BALB/c laboratory mice (purchased from a commercial supplier) without anaesthesia (Parasuraman et al. 2010). Blood cells were counted using a Nihon Kohden MEK-5208K cell counter (Nihon Kohden Corporation, Tokyo, Japan). Whole blood was stored in polypropylene tubes at room temperature and used for the measurement within two hours (Kaever et al. 1992), as long as the control chemiluminescence remained constant.

\section{Phagocyte activity measurement}

Phagocyte respiratory burst (RB) activity was measured using chemiluminescence enhanced by luminol (Sigma-Aldrich Merck KGaA, Darmstadt, Germany) (Papezikova et al. 2016). The reaction mixture included the ca $120 \mu \mathrm{l}$ blood sample diluted at a ratio of 1:50 in Hank's balanced salt solution, luminol dissolved in borate buffer and $0.25 \mathrm{mg} \cdot \mathrm{ml}^{-1}$ of Zymosan A (Sigma-Aldrich Merck KGaA) opsonised with bat serum as an activator. Chemiluminescence kinetics were measured at two different temperatures using a Cytation 3M reader (BioTek Instruments, Inc., Winooski, VT, USA) over two hours. The measuring temperatures were set at $25^{\circ} \mathrm{C}$ and $38^{\circ} \mathrm{C}$ to simulate variability in the physiological state of bats arousing and re-warming from hibernation torpor to homeothermy. The body surface temperature of bats at the time of blood sampling was approximately $24{ }^{\circ} \mathrm{C}$ (Pikula et al. 2017), indicating a much higher core body temperature (Bartonicka et al. 2017).

Five parameters characterising phagocyte activity were obtained from the data. The peak in the chemiluminescence curve (Peak) was measured in relative light units (RLU), representing maximum RB intensity. Total phagocyte activity (Integral) was defined as the reaction curve area (calculated directly from the raw data by the luminometer), with this value further adjusted to the total white blood cell count (Adjusted Integral). As individual phagocyte activity curves displayed different patterns, we also evaluated two time-related parameters (in seconds), i.e. time-to-start $\left(\mathrm{T}_{\text {start }}\right)$ and time-to-peak $\left(\mathrm{T}_{\text {peak }}\right)$, these reflecting the time necessary for the chemiluminescence curve to start growing and for phagocyte activity to reach its highest chemiluminescence value. $\mathrm{T}_{\text {start }}$ was defined as the first chemiluminescence measurement with a value over 1 , or as the point at which the equation reached value 1 . Time-to-end $\left(\mathrm{T}_{\text {end }}\right)$ reflected the time needed for the curve to reach its endpoint, i.e. the theoretical period during which all phagocyte activity was exhausted. This value was calculated mathematically from the defined equation (see below) and expressed against the point at which chemiluminescence declined to value 1 .

\section{Statistical analysis}

Normal distribution of variables was tested using the Kolmogorov-Smirnov and Shapiro-Wilk tests. As the variables were not normally distributed, and logarithmic (log) transformation did not result in normality, 
statistical analysis was conducted using the non-parametric Wilcoxon matched pair test and the Spearman rank order correlation. Interquartile range divided by the median, analogous to the coefficient of variation, was used as a measure of relative variability in the subsamples. Suitability of mathematical models (Sutton 1997) proposed for phagocyte activity evaluation was tested using the Akaike information criterion (AIC). Statistical analysis was performed in Statistica for Windows v. 13.3 or in R software using the support package $d p l y r$ (Wickham et al. 2019). Values of $P<0.05$ and $P<0.01$ were considered significant and highly significant, respectively, for all tests.

\section{Ethics statement}

Blood samples were collected from both bats and laboratory mice non-lethally while minimising animal distress, as stipulated by Czech Law No. 114/1992 on Nature and Landscape Protection (permits 1662/MK/2012S/00775/ MK/2012 and 00356/KK/2008/AOPK issued by the Agency for Nature Conservation and Landscape Protection of the Czech Republic) and the Ethics Committee of the Czech Academy of Sciences (No. 169/2011). The authors of the study are approved as competent to design and perform animal experiments and handle free-living bats (Czech Certificate No. CZ01341, §15d/3 of Act No. 246/1992).

\section{Results}

\section{Comparison of respiratory burst evaluations using mathematical} and mechanistic approaches

A mechanistic evaluation of RB proved relatively simple as we obtained the parameter values directly from the moving average datasets. For statistical evaluation, we first tested which mathematical model would best fit the data. Initially, we tried an empirical model with log-normal distribution, based on the equation:

$$
y=a x^{2} \exp ^{-b x}
$$

This simple dependence equation with its quadratic onset and exponential decrement turned out to be insufficient for describing real relationships. We then tried a variant using a generalised exponent:

$$
a x^{2} \exp ^{-b x^{p}}
$$

The Akaike information criterion confirmed that the second model with a generalised exponent was a better fit to the data in all datasets (Table 1). The equation was then transformed in order to calculate time-related or quantitative parameters, i.e.

$$
\frac{m}{t^{2}} x^{2} \exp \left(-\frac{2}{p}\left|\frac{x}{t}\right|^{p-1}\right)
$$

where $m$ is the peak of the function, $t$ is the time when the peak occurred and $p$ is the exponential power. This made it possible to determine all phagocyte activity parameters directly from the curves for each dataset. Other parameters, such as the time when the curve started to grow $\left(\mathrm{T}_{\text {start }}\right)$ and started to decline toward the end point $\left(\mathrm{T}_{\text {end }}\right)$, were calculated by making the equation equal to 1 (Fig. 1).

Both phagocyte activity evaluation approaches gave comparable results for the parameters measured (Peak, $\mathrm{T}_{\text {peak }}, \mathrm{T}_{\text {start }}$ ) in four of six dataset combinations (two bat species and two measurement temperatures; Fig. 2). Exceptions caused by extremely high variability in the original data (Fig. 1) were only found for greater mouse-eared bats $\left(\mathrm{T}_{\text {peak }}\right.$ at $25^{\circ} \mathrm{C} ; \mathrm{Z}=2.04$ $P=0.04 ; \mathrm{T}_{\text {start }}$ at $\left.38^{\circ} \mathrm{C} \mathrm{Z}=2.93, P=0.003\right)$. The values given by both approaches were significantly correlated $(P<0.05)$ in all parameters. The Integral and Adjusted Integral were excluded from this analysis due to the differing nature of the data produced by the mechanistic and mathematical approaches. 

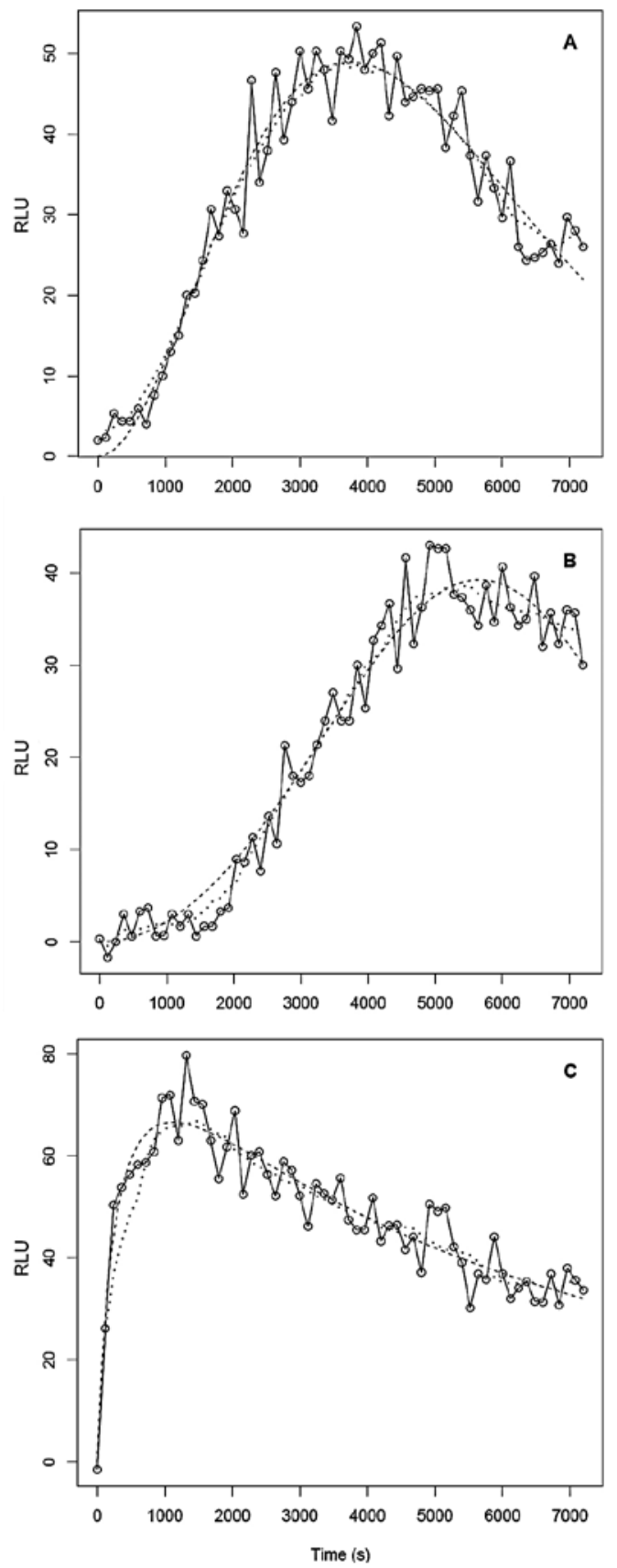

Fig. 1. Representative phagocyte activity kinetics for heterothermic bats Myotis myotis (A) and Nyctalus noctula (B) and the homeothermic laboratory mouse Mus domesticus (C). Activation is in RLU (reactive light units) and represents the intensity of respiratory burst over time (in seconds) at a measurement temperature of $25^{\circ} \mathrm{C}$. The three curves represent 1) raw data (dots connected by full line), 2) moving averages (dotted curve), and 3) calculated values (dashed curve). 

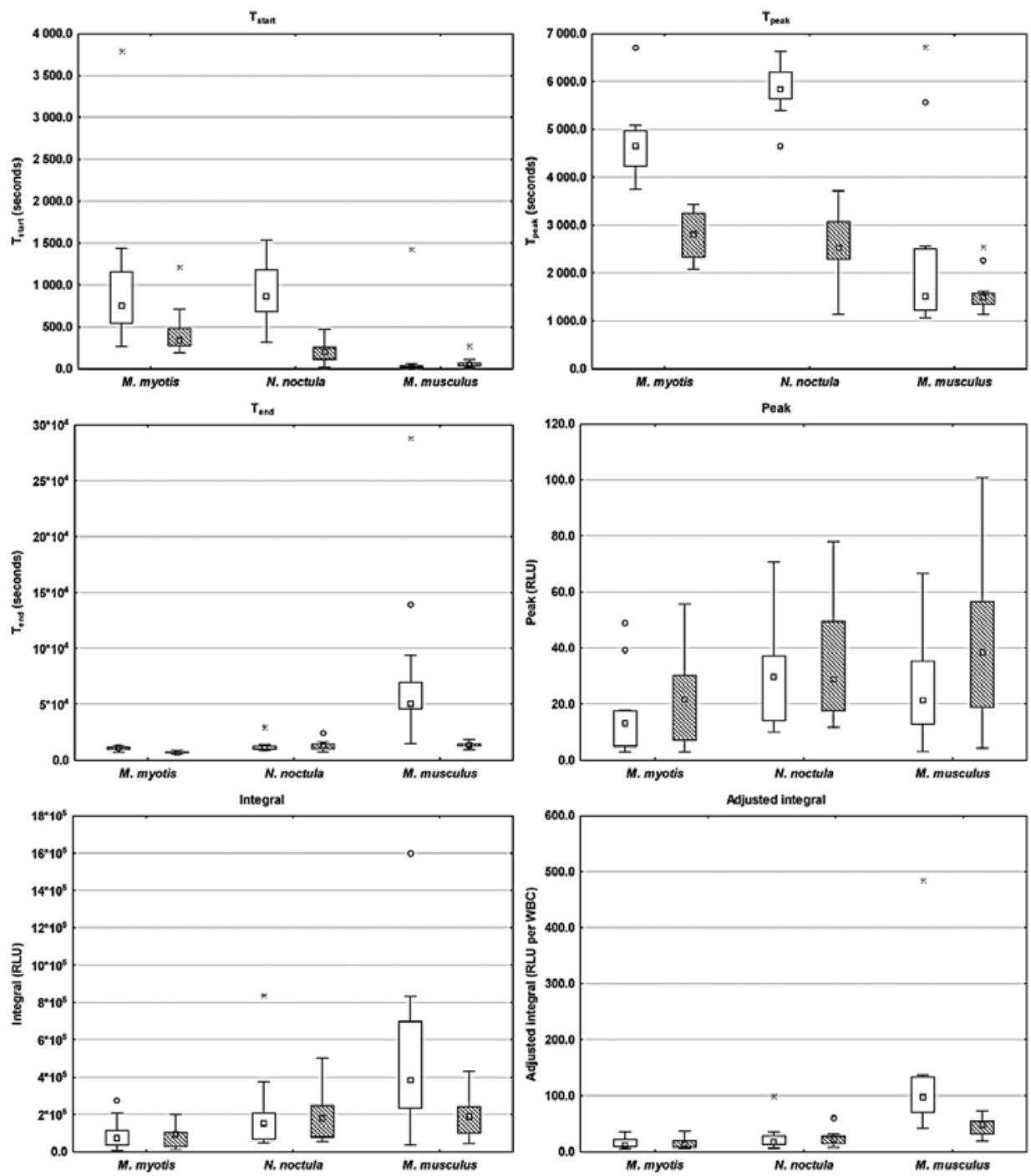

Fig. 2. Values of phagocyte activity parameters measured at two different temperatures. Explanation: empty boxes $=$ measurement temperature of $25^{\circ} \mathrm{C}$; dashed boxes $=$ measurement temperature of $38^{\circ} \mathrm{C}$; middle point $=$ median; box $=$ inter-quartile range; whiskers $=$ non-outlier range; dots $=$ outliers; stars $=$ extremes.

Influence of measurement temperature and model species on respiratory burst

Time-related parameters $\left(\mathrm{T}_{\text {start }}, \mathrm{T}_{\text {peak }}\right.$, and $\left.\mathrm{T}_{\text {end }}\right)$ of bat phagocyte activity were significantly shorter at the higher measuring temperature (Table 2). There was no difference in the Integral and Adjusted Integral measurements of total phagocyte activity (Table 2). The Peak value was again higher at the higher measuring temperature (Table 2). The lower measuring temperature had a much greater impact on phagocyte activity in the homeothermic laboratory mice (Fig. 2, Table 3). Peak and total phagocyte activity were both reduced along with all 
Table 1. Akaike information criteria for two mathematical models proposed for fitting phagocyte respiratory burst data.

\begin{tabular}{lcc}
\hline $\begin{array}{l}\text { Experiment } \\
\text { Species/cell incubation temperature }\end{array}$ & AIC basic & AIC advanced \\
\hline Myotis myotis $25{ }^{\circ} \mathrm{C}$ & 300.88 & 291.79 \\
Myotis myotis $38^{\circ} \mathrm{C}$ & 331.30 & 308.93 \\
Nyctalus noctula $25^{\circ} \mathrm{C}$ & 312.34 & 289.92 \\
Nyctalus noctula $38^{\circ} \mathrm{C}$ & 352.41 & 334.63 \\
Mus domesticus $25^{\circ} \mathrm{C}$ & 343.41 & 306.41 \\
Mus domesticus $38^{\circ} \mathrm{C}$ & 352.65 & 312.84 \\
\hline
\end{tabular}

The two models proposed for phagocyte activity evaluation (an empirical model with log-normal distribution and a model with a generalised exponent) were tested using the Akaike information criterion (AIC).

Table 2. Comparison of phagocyte activity parameters measured at two different temperatures. Significant results are in italics.

\begin{tabular}{lccccccccc}
\hline & \multicolumn{3}{c}{ Myotis myotis } & \multicolumn{3}{c}{ Nyctalus noctula } & \multicolumn{3}{c}{ Mus domesticus } \\
Time parameters & $\mathrm{n}$ & $\mathrm{Z}$ & $P$ & $\mathrm{n}$ & $\mathrm{Z}$ & $P$ & $\mathrm{n}$ & $\mathrm{Z}$ & $P$ \\
\hline $\mathrm{T}_{\text {peak }} 25 \& \mathrm{~T}_{\text {peak }} 38$ & 11 & 2.934 & 0.003 & 12 & 3.0594 & 0.002 & 14 & 0.910 & 0.363 \\
$\mathrm{~T}_{\text {start }} 25 \& \mathrm{~T}_{\text {start }} 38$ & 11 & 2.934 & 0.003 & 12 & 3.059 & 0.002 & 14 & 2.417 & 0.016 \\
$\mathrm{~T}_{\text {end }} 25 \& \mathrm{~T}_{\text {end }} 38$ & 11 & 2.934 & 0.003 & 12 & 0.784 & 0.433 & 14 & 3.296 & 0.001 \\
\hline Quantitative parameters & $\mathrm{n}$ & $\mathrm{Z}$ & $P$ & $\mathrm{n}$ & $\mathrm{Z}$ & $P$ & $\mathrm{n}$ & $\mathrm{Z}$ & $P$ \\
\hline Peak 25 \& Peak 38 & 11 & 2.401 & 0.016 & 12 & 2.197 & 0.028 & 14 & 3.296 & 0.001 \\
Integral 25 \& Integral 38 & 11 & 0.978 & 0.328 & 12 & 0.863 & 0.388 & 14 & 3.233 & 0.001 \\
Adjusted Integral 25 \& 38 & 11 & 0.622 & 0.534 & 12 & 0.628 & 0.530 & 13 & 3.180 & 0.001 \\
\hline
\end{tabular}

$\mathrm{T}_{\text {peak }}$ - time necessary for the chemiluminescence curve and for phagocyte activity to reach its highest chemiluminescence value; $\mathrm{T}_{\text {start }}$ - first chemiluminescence measurement with a value over 1 , or as the point at which the equation reached value $1 ; \mathrm{T}_{\text {end }}$ - time needed for the curve to reach its endpoint, i.e. the theoretical period during which all phagocyte activity was exhausted; Peak - maximum RB intensity; Integral - reaction curve area calculated directly from the raw data by the luminometer; Adjusted Integral - value further adjusted to the total white blood cell count

Table 3. Coefficient of variation (interquartile range divided by median) used as a measure of relative variability in subsamples. Increases in variability at low measurement temperatures are in italics.

\begin{tabular}{lcccccc}
\hline \multirow{2}{*}{ Parameter } & \multicolumn{2}{c}{ Myotis myotis } & \multicolumn{2}{c}{ Nyctalus noctula } & \multicolumn{2}{c}{ Mus domesticus } \\
& $38^{\circ} \mathrm{C}$ & $25^{\circ} \mathrm{C}$ & $38^{\circ} \mathrm{C}$ & $25^{\circ} \mathrm{C}$ & $38^{\circ} \mathrm{C}$ & $25^{\circ} \mathrm{C}$ \\
\hline $\mathrm{T}_{\text {start }}$ & 0.64 & 0.82 & 0.76 & 0.58 & 0.87 & 1.56 \\
$\mathrm{~T}_{\text {peak }}$ & 0.33 & 0.16 & 0.31 & 0.10 & 0.16 & 0.85 \\
$\mathrm{~T}_{\text {end }}$ & 0.16 & 0.20 & 0.33 & 0.27 & 0.19 & 0.47 \\
Peak & 1.08 & 0.99 & 1.11 & 0.78 & 0.99 & 1.07 \\
Integral & 0.84 & 1.14 & 0.95 & 0.91 & 0.76 & 1.23 \\
Adjusted Integral & 0.97 & 1.25 & 0.68 & 0.93 & 0.51 & 0.67 \\
\hline
\end{tabular}

$\mathrm{T}_{\text {start }}$ - first chemiluminescence measurement with a value over 1 , or as the point at which the equation reached value $1 ; \mathrm{T}_{\text {peak }}$ - time necessary for the chemiluminescence curve and for phagocyte activity to reach its highest chemiluminescence value; $\mathrm{T}_{\text {end }}$ - time needed for the curve to reach its endpoint, i.e. the theoretical period during which all phagocyte activity was exhausted; Peak - maximum RB intensity; Integral - reaction curve area calculated directly from the raw data by the luminometer; Adjusted Integral - value further adjusted to the total white blood cell count 
kinetic parameters, with the exception of $\mathrm{T}_{\text {peak }}$. Parameter variability in homeothermic mice increased dramatically at $25^{\circ} \mathrm{C}$.

\section{Discussion}

Comparison of respiratory burst evaluations using mathematical and mechanistic approaches

Considering the mechanistic evaluation of $\mathrm{RB}$, moving average is used for smoothing raw data with extremely high variability (here based on 11 values, including five samples before and five after each measurement) and is commonly used with time-series data to smooth short-term fluctuations and highlight longer-term trends or cycles (Chou 1989). In our case, the moving average could not be calculated with the first and final measurements; hence, they were excluded from further analysis. Moreover, the remaining first four and last four measurements had lower numbers of values averaged, i.e. 3, 5, 7 and 9 , respectively.

The proposed mathematical model also had its drawbacks. First, as expected, data variability was sometimes too high, which caused the prediction to fail. Second, in some cases, the predicted curve did not find the $\mathrm{T}_{\text {peak }}$ value because the last values measured did not always decrease. This problem could be fixed by making the measurement period longer, allowing full depletion of phagocyte capacity. Third, if phagocyte activity was around zero then the model could not intersperse the data with the required parameters. Nevertheless, the proposed equation and selected oxidative burst parameters offers a simple and reliable tool for comparison of phagocyte activity data. For example, the chemiluminescence reader measured the Integral directly from the raw data (the reaction curve area), but the values calculated covered the whole area under the theoretical curve obtained from data with the control subtracted. However, the equation models the theoretical course of phagocyte activity after the end of laboratory measurement and we expected that the area under the calculated curve would better reflect the total phagocyte activity of the individual. Consequently, only values calculated by the new equation were used for subsequent analysis.

Influence of measurement temperature and model species on respiratory burst

Low body temperatures are known to induce a reduction in the number of circulating phagocytes and their activity (Bouma et al. 2010a). Several studies have demonstrated that leukopaenia at low temperatures is not restricted to hibernating species only (Wilson et al. 1958; Ainsworth et al. 1991; Collazos et al. 1994). Here, by using the luminometric method which allows assessment of phagocyte metabolic activity at low whole blood volumes in small mammals (Lojek et al. 1997) and parameters characterising phagocyte activity, we were able to describe reaction kinetics and quality at defined temperatures in two heterotherm and one homeotherm species.

The bat phagocyte activity was faster at higher measuring temperatures, which corresponded with the euthermic state of bats, where the immune system is entirely active and quickly engages in defence reactions. Delayed kinetics might be expected in heterotherm bats as most physiological processes and the metabolism are reduced at the lower temperatures prevalent during hibernation and/or torpor bouts. Likewise, Marnila et al. (1995) showed that leukocytes from cold-acclimatised frogs $\left(5^{\circ} \mathrm{C}\right)$ were incapable of producing a RB at $37^{\circ} \mathrm{C}$. We assume that the innate immune parameters selected in this study will show different levels of immune response in hibernating and non-hibernating animals. The non-hibernating animals use daily torpor to survive rainy and cold weather so we expect this method to be applicable to them.

No difference in measurements of total phagocyte activity, indicating that all bat 
phagocytes are able to activate after a certain period, despite lower measuring temperatures. In this case, it follows that the time-related parameters would vary; however, somewhat surprisingly, the quantitative parameters did not.

Phagocyte activity of laboratory mice (representing the homeotherm species) was much more influenced by lower measuring temperature. We assume, therefore, that the optimal measuring temperature for the reaction corresponds to body temperature in homeotherms.

While both temperatures measured appear to be applicable for analysis of heterotherm phagocyte activity, parameters measured at $25{ }^{\circ} \mathrm{C}$ better reflect their physiological state during torpor and/or arousal from torpor. As the variability in phagocyte activity reflects the physiological and thermal state of particular animals, activity must be analysed separately for each specimen under natural conditions.

It may be assumed that the innate immune parameters achieve different response levels in hibernating and non-hibernating animals. As non-hibernating animals use daily torpor to respond to inclement weather (rain and cold), we expect the proposed method be also applicable during the active season of heterothermic animals.

To conclude, the proposed mathematical method for evaluating phagocyte activity is better suited for the analysis of innate immunity of hibernating and non-hibernating animals reflecting their body temperatures and physiological states.

\section{Acknowledgements}

This study was supported by the Czech Science Foundation (Grant No. 17-20286S). We are grateful to Dr. Kevin Roche for correction and improvement of the English text.

\section{References}

Ainsworth AJ, Dexiang C, Waterstrat PR, Greenway T 1991: Effect of temperature on the immune system of channel catfish (Ictalurus punctatus) - I. Leucocyte distribution and phagocyte function in the anterior kidney at $10{ }^{\circ} \mathrm{C}$. Comp Biochem Physiol 100: $907-912$

Andjus RK, Olivera M, Petrovic V, Rajevski V 1964: Influence of hibernation and of intermittent hypothermia on the formation of immune hemagglutinins in the ground squirrel. Ann Acad Sci Fenn Biol 71: 26-36

Bandouchova H, Bartonicka T, Berkova H, Brichta J, Kokurewicz T, Kovacova V, Linhart P, Piacek V, Pikula J, Zahradnikova A, Zukal J 2018: Alterations in the health of hibernating bats under pathogen pressure. Sci Rep 8: 6067

Bartonicka T, Bandouchova H, Berkova H, Blazek J, Lucan R, Horacek I, Martinkova N, Pikula J, Rehak Z, Zukal J 2017: Deeply torpid bats can change position without elevation of body temperature. J Therm Biol 63: 119-123

Bouma HR, Carey HV, Kroese FGM 2010a: Hibernation: the immune system at rest? J Leukoc Biol 88: 619-624

Bouma, HR, Dugbartey GJ, Boerema AS, Talaei F, Herwig A, Goris M, A. Buiten van A, Strijkstra AM, Carey HV, Henning RH, Kroese FGM 2013: Reduction of body temperature governs neutrophil retention in hibernating and nonhibernating animals by margination. J Leukoc Biol 94: 431-437

Bouma, HR, Strijkstra AM, Boerema AS, Deelman LE, Epema AH, Hut RA, Kroese FGM, Henning RH 2010b: Blood cell dynamics during hibernation in the European Ground Squirrel. Vet Immunol Immunopathol 136: 319-323

Boyer B, Barnes B 1999: Molecular and Metabolic Aspects of Mammalian Hibernation. Bioscience 49: 713-724

Buchtikova S, Simkova A, Rohlenova K, Flajshans M, Lojek A, Lilius EM, Hyrs1 P 2011: The seasonal changes in innate immunity of the common carp (Cyprinus carpio). Aquaculture 318: 169-175

Carey HV, Andrews MT, Martin SL 2003: Mammalian hibernation: cellular and molecular responses to depressed metabolism and low temperature. Physiol Rev 83: 1153-1181

Chou YL 1989: Statistical Analysis for Business and Economics. Elsevier Science, New York.

Collazos ME, Barriga C, Ortega E 1994: Enhanced granulocyte phagocytosis at low winter temperature and high summer temperature in the tench (Tinca tinca L.). Comp Biochem Physiol 109: 643-648

Havenstein N, Langer F, Stefanski V, Fietz J 2016: It takes two to tango: Phagocyte and lymphocyte numbers in a small mammalian hibernator. Brain Behav Immun 52: 71-80

Heldmaier G, Ortmann S, Elvert R 2004: Natural hypometabolism during hibernation and daily torpor in mammals. Respir Physiol Neurobiol 141: 317-329

Inkovaara P, Suomalainen P 1973: Studies on the physiology of the hibernating hedgehog. 18. On the leukocyte counts in the hedgehog's intestine and lungs. Ann Acad Sci Fenn Biol 200: 1-21

Kaever V, Robitzsch JT, Stangel WL, Schleinkofer L, Resch K 1992: Simultaneous detection of whole blood chemiluminescence in microtitre plates. Eur J Clin Chem Clin Biochem 30: 209-216 
Lojek A, Ciz M, Marnila P, Duskova M, Lilius EM 1997: Measurement of whole blood phagocyte chemiluminescence in the Wistar rat. J Biolum Chemilum 12: 225-231

Maniero GD 2002: Classical pathway serum complement activity throughout various stages of the annual cycle of a mammalian hibernator, the golden-mantled ground squirrel, Spermophilus lateralis. Dev Comp Immunol 26: 563-574

Marnila P, Tiiska A, Lagerspetz K, Lilius EM 1995: Phagocyte activity in the frog Rana temporaria: whole blood chemiluminescence method and the effect of temperature and thermal acclimation. Comp Biochem Physiol 111: $609-614$

Mondal S, Rai U 2001: In vitro effect of temperature on phagocytic and cytotoxic activities of splenic phagocytes of the wall lizard, Hemidactylus flaviviridis. Comp Biochem Physiol A-Mol Integr Physiol 129: 391-398

Murphy K, Travers P, Walport M, Janeway C 2012: Janeway's immunobiology. $8^{\text {th }}$ edn. Garland Science, New York

Papezikova I, Mares J, Vojtek L, Hyrsl P, Markova Z, Simkova A, Bartonkova J, Navratil S, Palikova M 2016 : Seasonal changes in immune parameters of rainbow trout (Oncorhynchus mykiss), brook trout (Salvelinus fontinalis) and brook trout $\times$ Arctic charr hybrids (Salvelinus fontinalis $\times$ Salvelinus alpinus alpinus). Fish Shellfish Immunol 57: 400-405

Parasuraman S, Raveendran R, Kesavan R 2010: Blood sample collection in small laboratory animals. J Pharmacol Pharmacother 1: 87-93

Pikula J, Bandouchova H, Kovacova V, Linhart P, Piacek V, Zukal J 2017: Reproduction of rescued vespertilionid bats (Nyctalus noctula) in captivity: veterinary and physiologic aspects. Vet Clin North Am Exot Anim Pract 20: $665-677$

Sutton PC 1997: Modelling population density with night-time satellite imagery and GIS. Comput Environ Urban Syst 21: 227-244

Wickham H, François R, Henry L, Müller K 2019: dplyr: A Grammar of Data Manipulation. R package version 0.8.0.1. https://CRAN.R-project.org/package=dplyr

Willson JT, Miller WR, Eliot TS 1958: Blood studies in the hypothermic dog. Surgery 43: 979-989 